\title{
Correction to: Skin cancer risk perception and sun protection behavior at work, at leisure, and on sun holidays: a survey for Danish outdoor and indoor workers
}

\author{
Kasper Grandahl ${ }^{1 *}$, Kristina Sophie Ibler ${ }^{2}$, Gunnar Hellmund Laier ${ }^{3}$ and Ole Steen Mortensen ${ }^{1,4}$
}

\section{Correction to: Environmental Health and Preventive Medicine (2018) 23:47 DOI: 10.1186/ \\ s12199-018-0736-x}

Following publication of the original article [1], it was highlighted that Table 3 contained an error. The $p$-value for use of long trousers and shirt with sleeves on sun holiday should not be preceded by a minus sign (-). The correct value is 231 . This Correction article shows the correct Table 3. The original article has been updated.

\footnotetext{
Author details

'The Department of Occupational Medicine, Copenhagen University Holbaek, Gl. Ringstedvej 4B, 4300 Holbaek, Denmark. ${ }^{2}$ The Department of Dermatology, Zealand University Hospital, Roskilde, Denmark. ${ }^{3}$ PFI (Production, Research, Innovation) Region Zealand, Sorø, Denmark. ${ }^{4}$ Section of Social Medicine, Department of Public Health, University of Copenhagen, Copenhagen, Denmark.
}

Received: 24 October 2018 Accepted: 24 October 2018

Published online: 14 November 2018

\section{Reference}

1. Grandahl K, et al. Skin cancer risk perception and sun protection behavior at

work, at leisure, and on sun holidays: a survey for Danish outdoor and indoor workers. Environ Health Prev Med. 2018;23:47. https://doi.org/10. 1186/s12199-018-0736-X.

\footnotetext{
*Correspondence: kagra@regionsjaelland.dk

${ }^{1}$ The Department of Occupational Medicine, Copenhagen University Holbaek,

Gl. Ringstedvej 4B, 4300 Holbaek, Denmark
} 


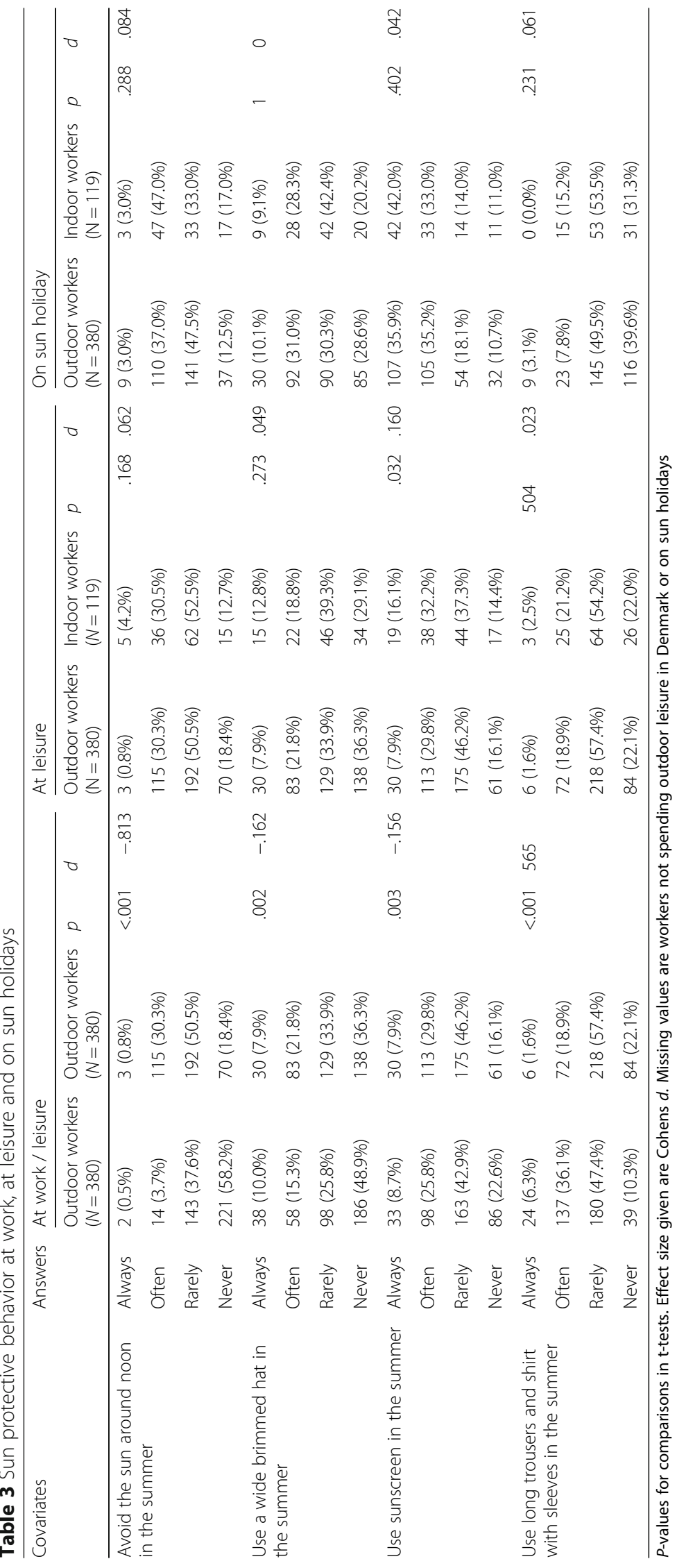

